The Astrophysical Journal, 602:892-903, 2004 February 20

(C) 2004. The American Astronomical Society. All rights reserved. Printed in U.S.A.

\title{
GLOBAL MAGNETOROTATIONAL INSTABILITY WITH INFLOW. I. LINEAR THEORY AND THE ROLE OF BOUNDARY CONDITIONS
}

\author{
Evy Kersalé, ${ }^{1}$ David W. Hughes, ${ }^{1}$ Gordon I. Ogilvie, ${ }^{2,3}$ Steven M. Tobias, ${ }^{1}$ and Nigel O. Weiss ${ }^{3}$ \\ Received 2003 July 4; accepted 2003 November 5
}

\begin{abstract}
We formulate a model system suitable for the systematic numerical investigation of global aspects of the magnetorotational instability and nonlinear dynamo action in accretion disks. The model consists of a cylindrical annulus occupied by an incompressible fluid with explicit viscosity and resistivity. Boundary conditions are imposed that permit an accretion flow appropriate to the stresses acting within the fluid to develop freely through the annulus. A steady basic state is identified in which a slow, steady accretion flow is driven by the explicit viscosity. We investigate the linear theory of this state subject to different choices of boundary conditions. The choice of boundary conditions is a crucial factor in determining the nature and growth rate of the instabilities. It is found that very rapidly growing wall modes occur generically, drawing energy artificially from outside the computational domain. However, by carefully selecting boundary conditions for which the total pressure is constrained at the radial boundaries, the wall modes are found to have growth rates bounded by the local properties of the magnetorotational instability. The resulting model provides the basis for a systematic exploration of nonlinear behavior in our future work.
\end{abstract}

Subject headings: accretion, accretion disks — instabilities - MHD

\section{INTRODUCTION}

Accretion disks are found around young stars, where they are the sites of planet formation; in interacting binary stars, where they surround compact stars or black holes; and in the centers of active galaxies, where they surround supermassive black holes. In these systems, orbiting gas can be accreted by the central object if its angular momentum is removed by a torque acting within or on the disk.

The magnetorotational instability (MRI) has been identified as a powerful source of angular momentum transport in accretion disks that are sufficiently ionized for the magnetic field to be dynamically coupled to the gas (Balbus \& Hawley 1998). Local numerical simulations of the nonlinear development of the MRI (Hawley, Gammie, \& Balbus 1995; Brandenburg et al. 1995; Stone et al. 1996; Matsumoto \& Tajima 1995) typically reach a state of three-dimensional magnetohydrodynamic (MHD) turbulence. This state is characterized by anisotropic Reynolds and Maxwell stress tensors that transport angular momentum outward and simultaneously sustain the turbulence by drawing energy from the large-scale shearing motion in the disk.

Numerous local studies have been carried out to investigate the implications of physical effects such as stratification (Miller \& Stone 2000), the Hall effect (Sano \& Stone 2002), radiation pressure (Turner, Stone, \& Sano 2002), and nonuniform electrical conductivity (Fleming \& Stone 2003). The shearing box model (Goldreich \& Lynden-Bell 1965; Hawley et al. 1995) has proven to be an invaluable tool for uncovering

\footnotetext{
${ }^{1}$ Department of Applied Mathematics, University of Leeds, Leeds LS2 9JT, UK; kersale@maths.leeds.ac.uk, dwh@maths.leeds.ac.uk, smt@maths. leeds.ac.uk.

${ }^{2}$ Institute of Astronomy, University of Cambridge, Madingley Road, Cambridge CB3 0HA, UK; gogilvie@ast.cam.ac.uk.

${ }^{3}$ Department of Applied Mathematics and Theoretical Physics, Centre for Mathematical Sciences, University of Cambridge, Wilberforce Road, Cambridge CB3 0WA, UK; n.o.weiss@damtp.cam.ac.uk.
}

the local physics of the MRI. It has the attractive property that a numerical calculation can be continued for many orbital periods and a statistically steady state attained. Owing to the symmetry of the shearing box, no further secular evolution occurs and no accretion flow develops.

More recently, global numerical simulations of the MRI have also been carried out for moderately thin disks or thick tori (e.g., Armitage 1998; Hawley 2000; Steinacker \& Papaloizou 2002; Machida, Hayashi, \& Matsumoto 2000). To the extent that any secular evolution occurs, these calculations generally have the nature of a run-down experiment (similar to the classical solution of the viscously spreading ring; Lynden-Bell \& Pringle 1974) since mass is permitted to leave the computational domain but has no source by which it may be replenished.

While informative, these studies leave a number of questions unanswered. Of particular interest is the relation between the MRI and the classical dynamo problem, in which one seeks fluid flows capable of sustaining a magnetic field against resistive decay (e.g., Moffatt 1978). The standard picture of dynamo action is that a complicated fluid motion, such as turbulent convection, initially causes an exponential amplification of a weak seed magnetic field. When the magnetic field attains a certain strength, the Lorentz force sufficiently modifies the flow such that no further amplification occurs, and the dynamo is saturated. An important distinction exists between a small-scale dynamo, in which magnetic energy is generated predominantly on length scales similar to those of the motion, and a large-scale dynamo, in which magnetic energy is imparted to structures of much larger scale.

However, the nature of dynamo action when the turbulence results from the MRI is different. Here the dynamo is always nonlinear (or nonkinematic, as noted by Hawley, Gammie, \& Balbus 1996) in the sense that the turbulent motion owes its origin to the magnetic field itself through the instability. This remarkable "bootstrapping" process merits further investigation as a novel paradigm of dynamo action. Saturation of the dynamo may then be related to the still unresolved issue of how 
the MRI saturates and what limits the turbulent stress that can be attained in an accretion disk. A further unanswered question is whether a large-scale dynamo also accompanies the MRI. This is of particular interest with regard to models of jets from accretion disks, which typically presume the existence of a largescale magnetic field threading the disk (e.g., Blandford \& Payne 1982; Shibata \& Uchida 1985, 1986).

In this paper and in future work, we undertake a new investigation of the MRI with the aim of addressing some of these outstanding questions. Our methodology differs from that of other authors. Rather than attempting to access a highly turbulent regime as close as possible to (and yet very remote from) the astrophysical one, we shall explore systematically a progression of complexity in the behavior of our model, with the aim of understanding the physical processes at work. Owing to the subtle nature of astrophysical dynamo action, where diffusion, although small, plays a vital role, an accurate control of dissipation is needed through the inclusion of explicit viscosity and resistivity.

This paper addresses the linear stability of a magnetized, accreting, Keplerian flow in a global, cylindrical model. Section 2 contains a detailed discussion of the model and the basic state, together with the governing equations and boundary conditions. A key feature of our model, discussed in $\S 3$, is the appearance of two distinct modes of instability, which we refer to as "body modes" and "wall modes." Section 4 discusses the origin of the wall modes via a simplified Cartesian model and demonstrates the significance of the choice of boundary conditions. In $\S 5$ we utilize the results of $\S 4$ in the cylindrical model. In $\S 6$ we discuss the implications of our results, particularly the vital role played by boundary conditions in models of accretion disks.

\section{SETUP OF THE MODEL}

\subsection{Modeling of the Disk}

In order to focus on the global development of the MRI, we adopt what is arguably the simplest model that retains all the essential features required for the linear and nonlinear study of the instability. We do not attempt to model the entire accretion disk but restrict attention to an annular section-localized far from the central object and the external regions - of a larger, extended disk (see Fig. 1). We consider a differentially rotating cylindrical annulus [with $r_{1}<r<r_{2}$ in cylindrical polar coordinates $(r, \varphi, z)]$ of finite thickness, composed of an incompressible, resistive, viscous plasma threaded by a magnetic field; in terms of the MRI, the assumption of

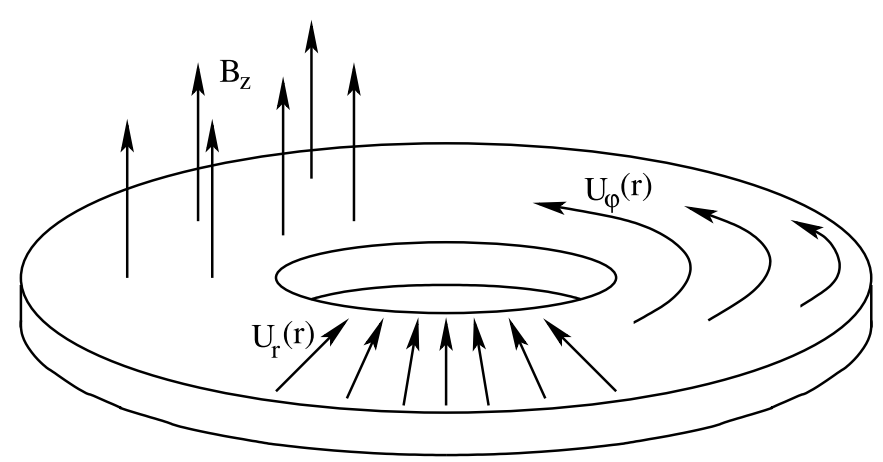

FIG. 1.-Sketch of our model of a slim, magnetized, dissipative accretion disk. A gravitational potential creates an azimuthal differentially rotating flow that, in turn, drives an accretion flow through viscous effects. incompressibility is not particularly restrictive. The dynamics of the dissipative disk relies on the gravitational potential due to the central mass $M *$; the self-gravity of the disk is supposed to be negligible. Furthermore, we do not take into account the influence of gravity in the direction transverse to the disk (which, for convenience, we shall refer to as the "vertical" direction). For consistency with the lack of vertical stratification we assume a small $H / r$ ratio, where $H$ and $r$ are the typical semithickness and radius of the disk, respectively; this requirement is satisfied simply by limiting the largest vertical scale of the modes developing in the slim cylinder (see $\S 2.4$ ). These simplifications exclude the effects of magnetic buoyancy (e.g., Terquem \& Papaloizou 1996), convection (e.g., Narayan et al. 2002), or the coupling of Rossby waves with density waves driven by a strong magnetic pressure (Tagger \& Pellat 1999), all of which, although possibly important, are not the concern of the current work.

One of the aims of our work is to compare a global cylindrical approach with that of the widely used local or shearing box approximation. The latter formulation models, in Cartesian geometry, a small sector of the disk; it removes any curvature effects but retains a linearized differential velocity together with the Coriolis force (Goldreich \& Lynden-Bell 1965). The main side effect of the shearing box model is its radial symmetry, which can only be broken by the curvature. Thus, even if, far from the central object, the curvature of the accretion disk is not an important issue in itself because it has a small contribution on the dynamics (for instance, Gammie \& Balbus 1994 show that density fluctuations have more relevance than curvature), it modifies significantly the symmetry of the models. Furthermore, in contrast to shearing box models, our global approach takes into account the vorticity gradient, which might be as important as the velocity gradient for the properties of turbulent motions.

Under the assumptions of our model, the governing equations for the velocity field $\boldsymbol{U}$ and magnetic field $\boldsymbol{B}$ are those of incompressible MHD, which may be written as

$$
\begin{gathered}
\frac{\partial \boldsymbol{U}}{\partial t}+(\boldsymbol{U} \cdot \nabla) \boldsymbol{U}=-\nabla \Phi-\frac{\nabla \Pi}{\rho}+\frac{1}{\mu_{0} \rho}(\boldsymbol{B} \cdot \nabla) \boldsymbol{B}+\frac{\mu}{\rho} \nabla^{2} \boldsymbol{U}, \\
\nabla \cdot \boldsymbol{U}=0 \\
\frac{\partial \boldsymbol{B}}{\partial t}+(\boldsymbol{U} \cdot \nabla) \boldsymbol{B}=(\boldsymbol{B} \cdot \nabla) \boldsymbol{U}+\frac{1}{\mu_{0} \sigma} \nabla^{2} \boldsymbol{B}, \\
\nabla \cdot \boldsymbol{B}=0,
\end{gathered}
$$

where $\rho$ is the (uniform) density of the disk, $\Phi=G M_{*} / r$ is the external gravitational potential acting on the disk, $\Pi=P+$ $B^{2} / 2 \mu_{0}$ is the total pressure (thermal plus magnetic), $\mu$ is the viscosity of the plasma, $\sigma$ is its electrical conductivity, and $\mu_{0}$ is the magnetic permeability.

To obtain a dimensionless representation of these equations, we scale lengths with $r_{1}$, the inner radius of the disk, times with $\Omega_{\mathrm{K}}^{-1}\left(r_{1}\right)$, where $\Omega_{\mathrm{K}}(r)=\left(G M_{*} / r^{3}\right)^{1 / 2}$ is the Keplerian frequency, and magnetic energy density with the rotational kinetic energy density $G M * \rho / r_{1}$. Equations (2) and (4) are clearly unchanged by any scaling; equations (1) and (3) become

$$
\frac{\partial \boldsymbol{U}}{\partial t}+(\boldsymbol{U} \cdot \nabla) \boldsymbol{U}=\nabla\left(\frac{1}{r}\right)-\nabla \Pi+(\boldsymbol{B} \cdot \nabla) \boldsymbol{B}+\nu \nabla^{2} \boldsymbol{U}
$$




$$
\frac{\partial \boldsymbol{B}}{\partial t}+(\boldsymbol{U} \cdot \nabla) \boldsymbol{B}=(\boldsymbol{B} \cdot \nabla) \boldsymbol{U}+\eta \nabla^{2} \boldsymbol{B},
$$

where $\nu$ and $\eta$ are, respectively, the dimensionless viscosity and magnetic diffusivity. In our scaled variables the disk extends from $r=1$ to $r=r_{2} / r_{1}=R$, say.

\subsection{The Basic Equilibrium State}

In the present work we are interested in the instability, to the MRI, of a steady, radially dependent disk. The system of equations (2), (4), (5), and (6) admits simple, time-independent, axisymmetric and $z$-invariant equilibrium solutions with no vertical motion and only a vertical magnetic field. These are governed by the following set of ordinary differential equations:

$$
\begin{gathered}
U_{r} \frac{d U_{r}}{d r}-\frac{U_{\varphi}^{2}}{r}=-\frac{1}{r^{2}}-\frac{d \Pi}{d r}+\nu\left[\frac{1}{r} \frac{d}{d r}\left(r \frac{d U_{r}}{d r}\right)-\frac{U_{r}}{r^{2}}\right] \\
U_{r} \frac{d U_{\varphi}}{d r}+\frac{U_{r} U_{\varphi}}{r}=\nu\left[\frac{1}{r} \frac{d}{d r}\left(r \frac{d U_{\varphi}}{d r}\right)-\frac{U_{\varphi}}{r^{2}}\right] \\
\frac{d}{d r}\left(r U_{r}\right)=0 \\
U_{r} \frac{d B_{z}}{d r}=\frac{\eta}{r} \frac{d}{d r}\left(r \frac{d B_{z}}{d r}\right) .
\end{gathered}
$$

The incompressibility condition given by equation (9) determines the radial velocity profile as

$$
U_{r}=\frac{\alpha}{r} .
$$

In our model, a nonzero radial flow is driven by the viscosity acting on the azimuthal component of the velocity. The latter is determined by integrating the azimuthal component of the momentum equation, to give

$U_{\varphi}= \begin{cases}\beta r^{(1+\alpha / \nu)}+\gamma / r & \text { if } 2+\alpha / \nu \neq 0 \\ (\beta \ln r+\gamma) / r & \text { otherwise. }\end{cases}$

The total pressure (for the case of $2+\alpha / \nu \neq 0$ ) is determined by the radial momentum balance,

$\Pi=\frac{1}{r}-\frac{\alpha^{2}+\gamma^{2}}{2 r^{2}}+\frac{\beta^{2}}{2(1+\alpha / \nu)} r^{2(1+\alpha / \nu)}+2 \frac{\beta \gamma \nu}{\alpha} r^{\alpha / \nu}+\delta$.

The magnetic field is obtained by integrating the vertical component of the induction equation, giving

$$
B_{z}=\lambda r^{\alpha / \eta}+B_{0}
$$

In the above equations, $\alpha, \beta, \gamma, \delta, \lambda$, and $B_{0}$ are constants of integration. If required, it is straightforward to add the azimuthal equilibrium magnetic field,

$$
B_{\varphi}=B_{1} r^{(-1+\alpha / \eta)}+\zeta r
$$

This simply induces a modification in the equilibrium pressure profile.
We are interested in weakly magnetized, cold, slim disks for which the leading order balance is between Keplerian rotation and the gravitational attraction of the central object. However, in an incompressible fluid a Keplerian rotation need not arise naturally since the gravitational force can, in principle, be compensated by a pressure gradient, with no further consequences. Thus, we impose a Keplerian rotation by the choice of parameters $\alpha=-3 \nu / 2, \beta=1$, and $\gamma=0$. For the bulk of the present work we consider disks threaded initially by a uniform vertical magnetic field $B_{z}=B_{0}$; i.e., we set $\lambda=0$. In $\S 6$ we consider briefly the role of an additional azimuthal field.

\subsection{Boundary Conditions}

As discussed in the introduction, probably the most important issue in this paper is the role of the boundary conditions. Since our model aims to describe only an annular section of a disk of much greater radial extent, we wish to impose the same boundary conditions at each radial boundary and also desire that these be as "neutral" as possible. Furthermore, and this is the most significant feature of our model, we wish to allow the accretion to evolve without undue constraint and so impose no boundary condition on the radial flow. Thus, our approach differs, for instance, from that adopted by Knobloch (1992) and Dubrulle \& Knobloch (1993), who impose impermeable radial boundary conditions, or Papaloizou \& Nelson (2003), who adapt their basic state in order to stabilize the MRI in the vicinity of the boundaries, or Curry \& Pudritz (1996), who suppose that the accretion disk is surrounded by a vacuum containing a potential magnetic field.

When viscous and resistive processes are taken into account, the system of equations of incompressible MHD (eqs. [2], [4], [5], [6]) is of 10th order with respect to the variable $r$, requiring the choice of 10 radial boundary conditions. In the case of ideal MHD (with $\nu=\eta=0$ ) the order of the system (in radius), and hence the number of radial boundary conditions required, drops to two.

For consistency with the imposed Keplerian profile of the basic state, we apply the following mixed boundary condition on the azimuthal velocity:

$$
\left.\frac{\partial}{\partial r} U_{\varphi}\right|_{1, R}=-\left.\frac{U_{\varphi}}{2 r}\right|_{1, R} .
$$

One way of avoiding the imposition of a boundary condition on $U_{r}$ is to impose a second boundary condition on $U_{\varphi}$, the most natural choice being to maintain the azimuthal velocity of the disk at its initial Keplerian value, i.e., to set

$$
\left|U_{\varphi_{1, R}}=\frac{1}{r^{1 / 2}}\right|_{1, R}=\text { const. }
$$

It turns out, however, as described in detail in $\S 3$, that the latter boundary condition allows not only the development of the MRI but also the more rapid development of a family of wall modes, concentrated near the radial boundaries. To reduce the impact of these modes, we have therefore considered an alternative boundary condition to equation (17), in which the total pressure is maintained at a (possibly different) constant value on the boundaries, i.e.,

$$
\left.\Pi\right|_{1, R}=\text { const. }
$$


Clearly equation (17) prevents rundown of the disk; except for a special choice of pressure boundary conditions, this is true for equation (18) also, since the gravitational force cannot be compensated by the pressure gradient. Equations (16) and (17) (or eq. [18]) are the conditions of significance. The remaining conditions, on $U_{z}$ and on two components of the field (the third following from $\nabla \cdot \boldsymbol{B}=0$ ), are chosen to be as "passive" as possible. Our choice, which is by no means the only acceptable one, is

$$
\begin{array}{r}
\left.\frac{\partial}{\partial r} U_{z}\right|_{1, R}=0, \\
\left.\frac{\partial}{\partial r} B_{z}\right|_{1, R}=0 \\
\left.\frac{\partial}{\partial r}\left(r B_{\varphi}\right)\right|_{1, R}=0 .
\end{array}
$$

For the ideal (nondissipative) system, for which we are allowed to impose only two boundary conditions, we employ either equation (17) or equation (18).

\subsection{The Linear Stability Problem}

The governing equations and boundary conditions discussed above are those describing the fully nonlinear evolution of the disk from its initial Keplerian state with an imposed vertical field. Here we are concerned only with the initial, linear development of the instability, deferring discussion of the nonlinear evolution to a subsequent paper. Following standard techniques, we consider the response of the equilibrium states defined in $\S 2.3$ to small-amplitude perturbations, governed by the linearized versions of the momentum and induction equations. Exploiting the fact that the basic state is steady and independent of $\varphi$ and $z$, we may express all perturbation variables in the normal mode form

$$
\xi(\boldsymbol{r}, t) \equiv \xi(r) \exp (s t+i m \varphi+i k z),
$$

where $\gamma=\Re(s)$ is the growth rate of the normal mode and $\omega=\Im(s)$ is its frequency. For positive values of the azimuthal wavenumber $m$, prograde and retrograde modes correspond, respectively, to negative and positive values of the frequency.

The set of governing linear equations, described in detail in the Appendix, may be expressed as a generalized eigenvalue problem

$$
s \mathcal{I}(r) \boldsymbol{\xi}(r)=\mathcal{L}(r) \boldsymbol{\xi}(r),
$$

where the eigenvalue is the complex coefficient $s=\gamma+i \omega$, the eigenvector $\xi$ consists of the perturbation variables, and $\mathcal{L}$ is the linear MHD operator. The operator $\mathcal{I}$ is the identity with respect to the velocity and magnetic field perturbations but the zero operator for the pressure perturbation. The eigenvalue problem is completed by specifying the linear version of the two different sets of boundary conditions described in $\S 2.3$.

For both sets the following conditions are applied:

$$
u_{\varphi}^{\prime}+\frac{u_{\varphi}}{2 r}=u_{z}^{\prime}=\left(r b_{\varphi}\right)^{\prime}=b_{z}^{\prime}=0 \quad \text { on } r=1, r=R,
$$

where, as in the Appendix, lowercase characters refer to the Eulerian perturbations and primes denote radial derivatives.
For the case where the azimuthal velocity is held constant on the boundaries, the perturbation velocity satisfies

$$
u_{\varphi}=0 \quad \text { on } r=1, r=R .
$$

For the alternative boundary condition, in which the total pressure is held constant, equation (25) is replaced by

$$
\pi=0 \quad \text { on } r=1, r=R .
$$

For the nondissipative case, for which only two boundary conditions are required, we impose either equation (25) or equation (26).

All of the results presented hereafter are for a disk of unit radial extent (i.e., $R=2$ ). It is important to note that there is no physical limitation to the thickness of the disk inherent in the equations; the consistency of the thin-disk approximation that we have made therefore relies on the imposition of a lower bound on the vertical wavenumber spectrum. Thus, to ensure a ratio of, for example, $H / r \sim \frac{1}{8}$, we have to consider modes with vertical wavelength smaller than 0.5 (assuming that half of the largest wavelength fits vertically in the disk). In terms of wavenumbers, only those greater than $k_{\min }=4 \pi \simeq$ 12.6 are relevant; this limit increases to $k_{\min } \simeq 31.4$ for a ratio of $H / r \sim 1 / 20$.

The linear system given by equation (23) has been discretized using fourth-order finite differences and has then been written as a matrix-form generalized eigenvalue problem. An inverse iteration algorithm has been used systematically to find the closest eigenvalue to an initial guess, together with the corresponding eigenvector; once a mode has been located for one set of parameter values, it can be tracked very easily throughout an extended parameter space. In addition, we have also used a shooting method (including a variable step size) for double-checking the results in those few cases where the finite difference version of the boundary conditions might have induced errors.

\section{DISTINGUISHING BODY MODES AND WALL MODES}

In this section we perform a preliminary survey of the linear stability properties of the basic Keplerian disk for the case when the differential rotation in the disk is enforced by imposing the azimuthal velocity at the boundaries, i.e., by applying the boundary condition given by equation (17) or, more precisely, its linear version (eq. [25]).

It is important to recall that the free energy source that feeds the unstable modes in accretion disks is the differential rotation, driven, in our model, by the action of the boundaries. Hence, it is reasonable to expect the normal modes to evolve on a timescale of the order of the inverse of the shear frequency, $|d \Omega / d \ln r|$, where $\Omega$ is the typical value of the angular velocity. For instance, for localized modes, in the absence of dissipation, the maximal growth rate is $\gamma_{\max }=$ $\frac{1}{2}|d \Omega / d \ln r|$ (Balbus \& Hawley 1998); i.e., for our choice of units, $\gamma_{\max }=0.75$ in the vicinity of the inner boundary. Therefore, any mode that possess a growth rate larger than this maximal value must be receiving energy from outside the disk.

In the next three subsections we examine both dissipative and nondissipative disks. In $\S 3.1$ we consider the special case of axisymmetric disturbances to a nondissipative disk, for which we find unstable MRI modes driven by processes occurring throughout the disk. We refer to such disturbances as body modes. Such modes can also be found for nonaxisymmetric 
perturbations and in dissipative disks; their properties are described in $\S 3.2$. Another class of unstable perturbations, which we term wall modes, are found if either nonaxisymmetry or dissipation is included. To be precise, we label growing disturbances as wall modes when the maximum of the perturbation to the radial velocity is localized on the boundary; their properties are discussed in $\S 3.3$.

\subsection{Axisymmetric, Ideal Body Modes}

We begin our investigation with the simplest case, that of a nondissipative disk subject to axisymmetric perturbations. This particular subclass of solutions to the linearized equation (23) is nongeneric in its behavior, largely as a result of the consequences of the boundary conditions. As noted earlier, the removal of dissipation reduces the number of boundary conditions from 10 to two; in this case we impose $u_{\varphi}=0$. The further assumption of axisymmetry determines the allowed values of the other perturbed variables on the boundaries. It follows that $u_{z}^{\prime}=\pi^{\prime}=b_{\varphi}=b_{z}^{\prime}=0$ and also more importantly $u_{r}=b_{r}=0$; i.e., the imposition of an azimuthal velocity immediately implies that the radial boundaries are impermeable at linear order. Thus, the disk is isolated from the outside in this particular limit. We shall see later that this means that wall modes (described in $\S 3.3$ ) are not permissible solutions for the system. Hence, unstable modes can only take the form of body modes, as described below.

Figure 2 shows contours of constant growth rate for the MRI body mode as a function of vertical wavenumber and magnetic field amplitude. The unstable mode exhibits the expected properties of the local MRI modes. The instability is suppressed by increasing magnetic tension (of magnitude $k B_{0}$ ); furthermore, for a given value of the magnetic field the wavenumber of the most unstable mode is close to that for which the mode is stabilized. However, in the nondissipative regime there is instability for any value of $k$, provided that $B_{0}$ is suitably chosen such that the product $k B_{0}$ is order unity, with the growth rate maximized by the value obtained in the local approximation. Indeed, in the limit of large wavenumber the unstable mode becomes localized (in the vicinity of the inner boundary, where the shear frequency reaches its highest value), and its growth rate converges to the maximum $\gamma_{\max }=0.75$; this occurs in the limit of infinitesimal magnetic field, as long as the instability is able to develop on infinitesimal scales. We also note that when both $k$ and $B_{0}$ are small, the disk is still unstable but with much smaller growth rates; indeed the restoring and destabilizing forces are then both weak. The properties of these modes are therefore very straightforward and can be understood within the confines of a local analysis.

\subsection{Body Modes in the General Case}

If the assumption of axisymmetry is relaxed for a nondissipative disk, then the imposed boundary condition $u_{\varphi}=0$ on the radial boundaries no longer implies that $u_{r}=0$, and the disk is no longer isolated. However, body modes with similar properties to the axisymmetric ideal modes can still be found as exponentially growing solutions to equation (23), as shown for $m=1$ in Figure $2 b$. Although the axisymmetric mode is the most unstable body mode that develops in a nondissipative disk, instability can be found for any value of the azimuthal wavenumber $m$, provided that $B_{0}$ is sufficiently weak. As $m$ increases, the stabilization of the mode always occurs for the same order of magnitude of the product $k B_{0}$, but the growth rate contours are shifted toward high values of $k$ and low values of $B_{0}$. Unlike the axisymmetric MRI modes, which undergo an exchange of stabilities, nonaxisymmetric modes are always overstable. The mathematical nature of the linear problem is also modified when dissipative processes occur in the disk; the order of the system, as well as the number of boundary conditions, increases from two to 10 . However, although none of these boundary conditions isolate the accretion disk from the outside and radial inflow/outflow at the boundaries is allowed, our choice nonetheless allows a clearly identified dissipative counterpart to the unstable ideal body modes.

Figure $3 a$ demonstrates the typical reduction of the domain of instability of the axisymmetric body modes in $k-B_{0}$ space when dissipative effects are taken into account. This figure shows strong similarities to the low $k$, high- $B_{0}$ regime of its ideal counterpart (Fig. 2a), but the dissipation, acting on both the velocity field and the magnetic field, can be seen to have two main effects. Dissipation tends to damp body modes and

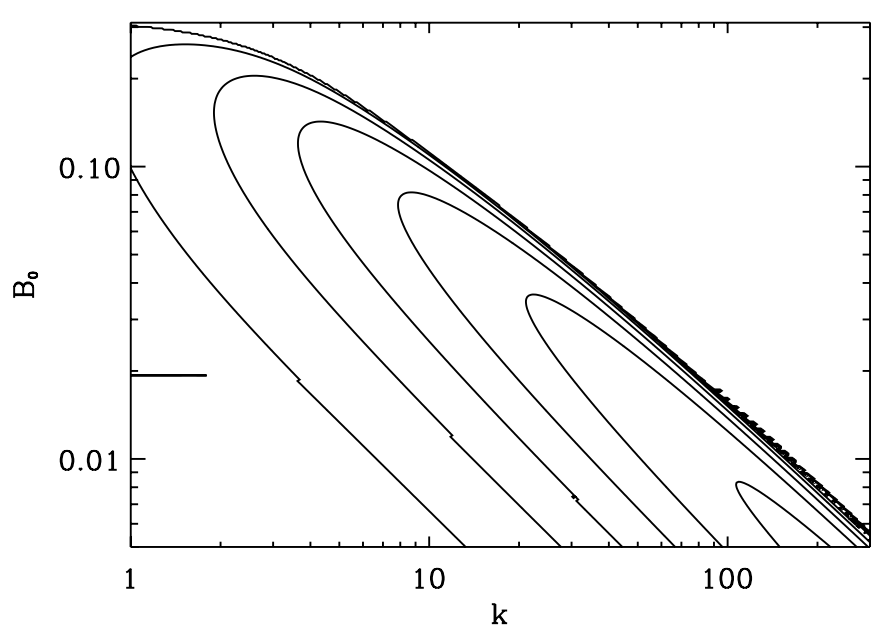

FIG. $2 a$

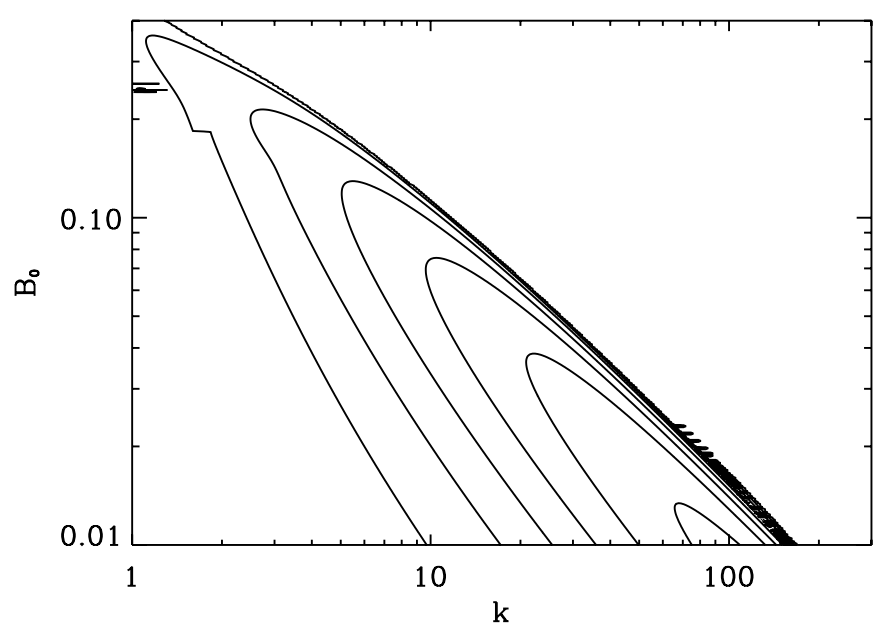

FIG. $2 b$

FIG. 2.-Contour plot in $k$ - $B_{0}$ parameter space of the growth rate of the most unstable mode for a nondissipative disk. $(a)$ Axisymmetric body mode $(m=0)$;

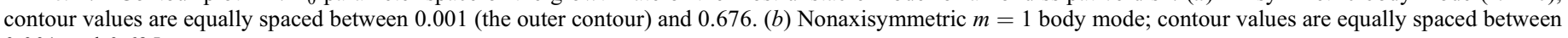
0.001 and 0.635 


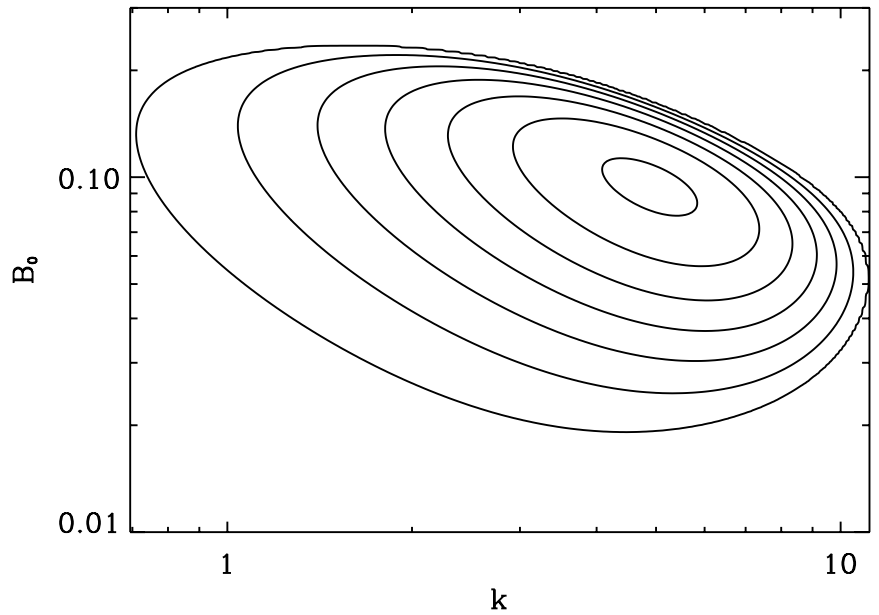

FIG. $3 a$

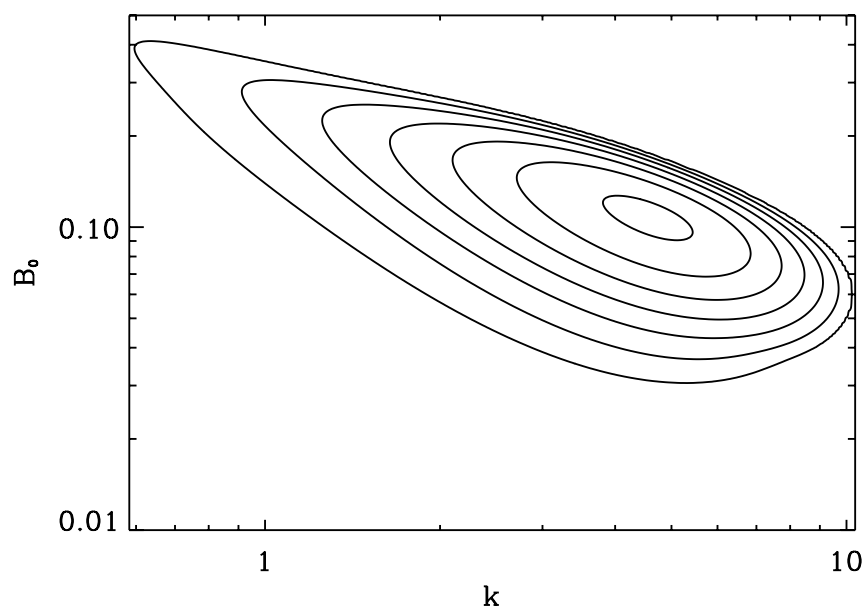

FIG. $3 b$

FIg. 3. - Contour plot in $k-B_{0}$ parameter space of the growth rate of the most unstable mode in a dissipative disk with $\nu=\eta=0.003$. (a) Axisymmetric body mode: this mode is nonoscillating and attains its maximal growth rate $\gamma=0.215$ for $B_{0}=0.094$ and $k=4.9$; contours are evenly spaced between 0.001 and 0.208 . (b) Nonaxisymmetric $m=1$ body mode: this mode reaches its maximal growth rate $\gamma=0.177$ for $B_{0}=0.108$ and $k=4.6$ with frequency $\omega=-0.616$; contours are evenly spaced between 0.001 and 0.172 .

reduce their growth rates over the entire instability domain; in particular, the low- $k$, low- $B_{0}$ regime is stabilized. Resistivity and viscosity also induce a dissipative cutoff for the highest values of $k$ and $m$ in the unstable body mode spectrum. The form of the eigenfunction for an axisymmetric body mode is shown in Figure $4 a$, where the velocity perturbations can be seen to take their maximal values in the body of the disk. Note that for this axisymmetric mode, nodes may appear in the modulus of the eigenfunctions.

A similar picture is found for nonaxisymmetric $(m=1)$, nondissipative body modes, as shown by the growth rate contours in Figure $3 b$. Here the maximal growth rate is a little lower than for the corresponding axisymmetric mode, but a large range of unstable wavenumbers and field strengths is available. A typical eigenfunction of such an unstable mode is shown in Figure $4 b$. For this particular case, no nodes are found in the eigenfunction, but radial structure is apparent. As the azimuthal wavenumber $m$ is increased further, more spatial oscillations are observed as the radial length scale of the eigenfunction decreases.

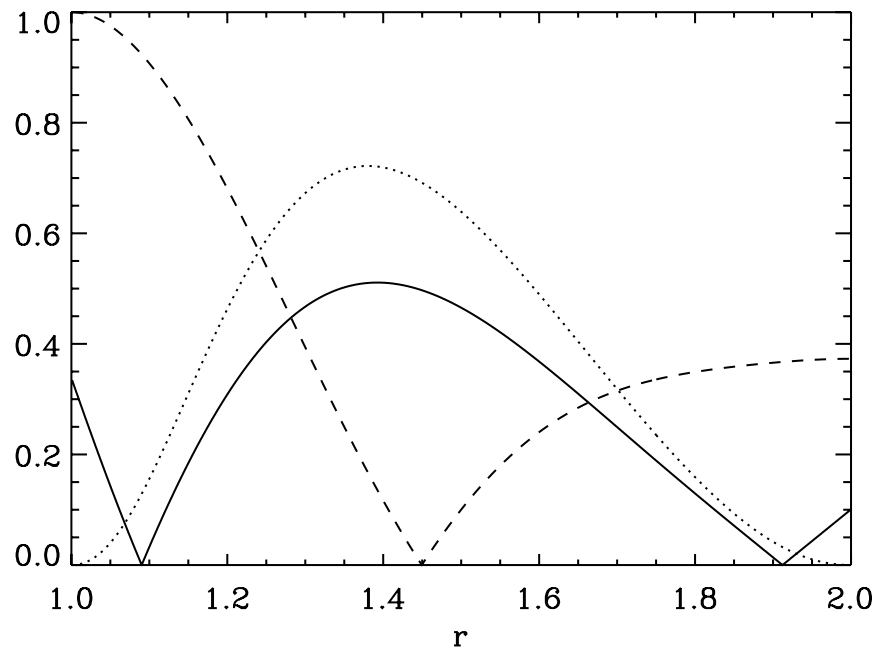

FIG. $4 a$
Figure 5 demonstrates a useful way of determining the mode of maximum growth rate as a function of wavenumber and field amplitude (in this case for a dissipative axisymmetric mode). The solid line represents the growth rate of the most unstable mode for a given field strength $B_{0}$, while the dashed line gives the corresponding wavenumber. From this graph it is easy to determine that the fastest growing mode occurs for an applied field strength $B_{0}=0.0346$ with corresponding wavenumber $k=20.8$. This type of plot will be very useful as a guide to simulations in the nonlinear regime.

\subsection{Wall Modes}

We have shown that, as expected, MRI body modes are natural solutions of the system in all cases. In this section we shall demonstrate that, so long as radial flows are not prevented by the boundary conditions, another class of modes are unstable: these we term wall modes. As noted in $\S 3.1$, the boundary conditions for axisymmetric, nondissipative modes preclude the possibility of a radial flow in the perturbation velocity at the boundary, and so we do not find wall modes for

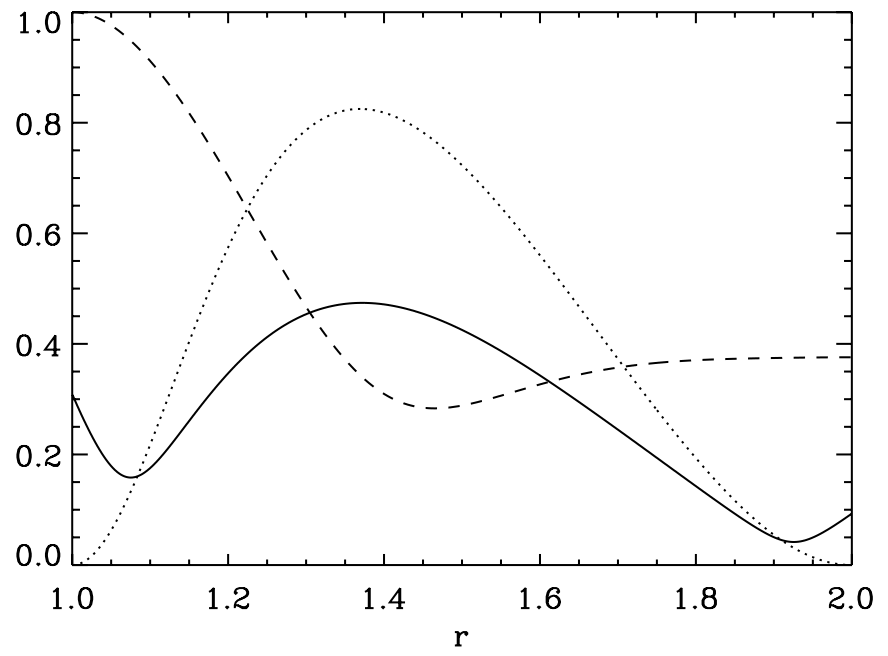

FIG. $4 b$

FIG. 4. - Eigenfunctions corresponding to the modes of maximum growth rate shown in Fig. 3. The moduli of the linear perturbations $u_{r}, u_{\varphi}$, and $b_{z}$ are plotted in solid, dotted, and dashed lines, respectively. 


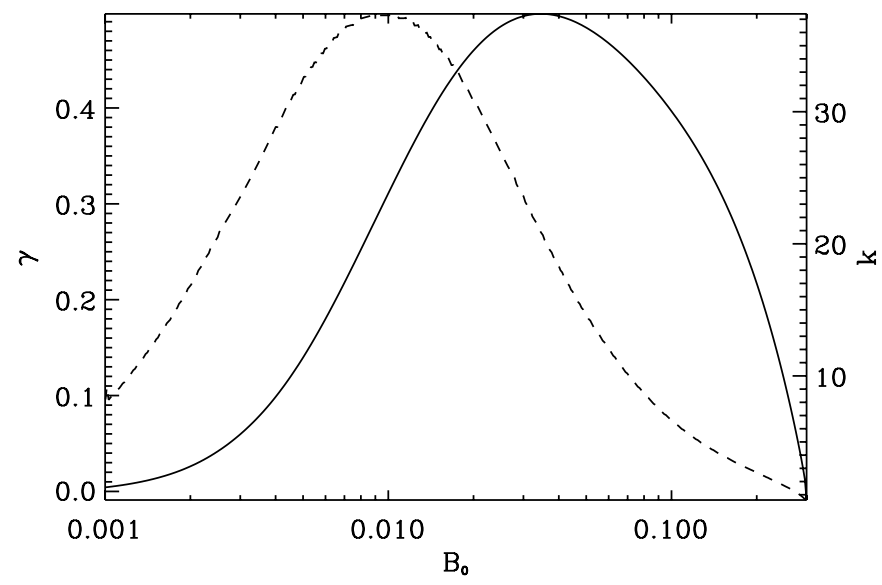

FIG. 5.-Growth rate (solid line) and corresponding wavenumber (dashed line) of the mode of maximum growth rate as a function of $B_{0}$ for an axisymmetric body mode with $\nu=\eta=1 \times 10^{-4}$. The most unstable mode is nonoscillating with growth rate $\gamma=0.4982$, obtained for $k=20.8$ and $B_{0}=0.0346$

this case. If, however, the system is such as to allow a radial flow at the boundary, and this may occur in either dissipative or nonaxisymmetric, nondissipative systems, then wall modes can develop.

Figure 6 illustrates an example of this phenomenon, showing the eigenfunctions for a nonaxisymmetric $(m=1)$, nondissipative wall mode, which has developed on the inner boundary of the disk. In general, wall modes may develop on either of the radial boundaries, but they are not symmetric, with the wall mode on the outer radial boundary having a smaller growth rate than that attached to the inner boundary. Interestingly, extending the cylindrical domain in radius leads to the eventual suppression of the wall mode on the outer boundary, the growth rate tending to zero as $R \rightarrow \infty$. However, the extension of the domain has little effect on the wall mode developing on the inner radial boundary.

The presence of wall modes as global solutions is not unexpected, given that the local shear frequency in a Keplerian disk reaches its maximum value on the inner boundary.

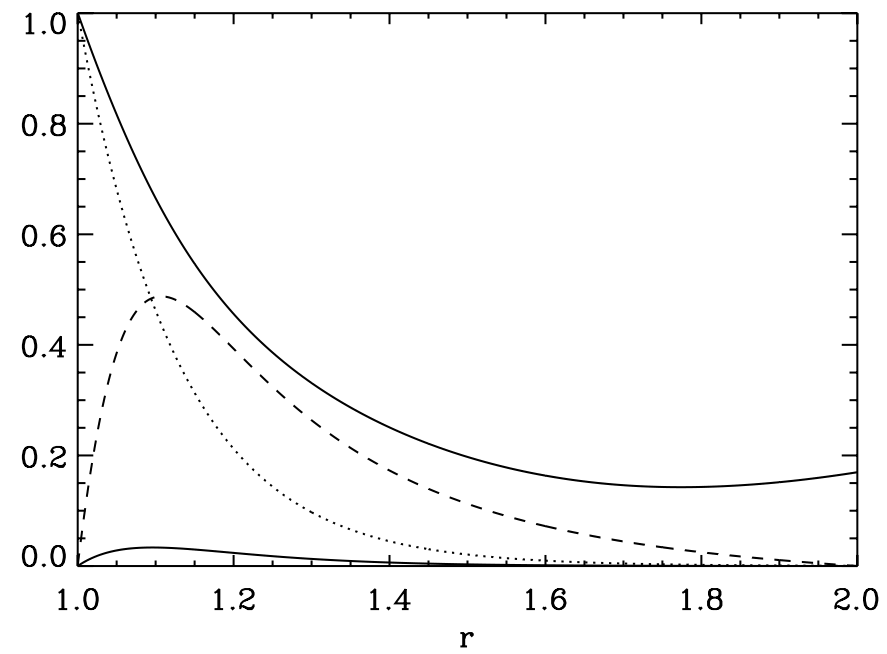

FIG. 6.-Eigenfunction of the wall mode with $m=1, \nu=\eta=0$. The solid and dashed lines show the moduli of the perturbations of $u_{r}$ and $u_{\varphi}$, respectively, for $k=2$; the growth rate of this mode is $\gamma=0.303$. The dotted and dot-dashed lines show the moduli of the perturbations of $u_{r}$ and $u_{\varphi}$, respectively, for $k=7$; the growth rate is $\gamma=1.117$.
However, the behavior of their growth rate is surprising, as shown in Figure 7. Here the growth rate is plotted as a function of wavenumber for different values of the imposed vertical field strength. For the modes with no dissipation, the growth rate is a monotonically increasing function of both $k$ and $B$ for the range of parameter values that we have investigated. There does not appear to be any maximum to the growth rate attainable, and, consequently, growth rates well in excess of the theoretical maximum available from the local shear energy $(\gamma=0.75)$ are found. In contrast to the body modes, magnetic tension does not appear to lead to a stabilization of these wall modes. The behavior of dissipative modes is similar. Again these modes show an increasing growth rate with applied magnetic field and an initial increase of growth rate with wavenumber. For large enough wavenumbers, however, the effects of dissipation are able to suppress the formation of arbitrarily small scales and the wall mode is damped. The maximum growth rate of these wall modes is, however, large (and can be made even larger by increasing the applied field $B_{0}$ ) compared with the maximum of the corresponding body mode. This result is both puzzling and disturbing. What is the origin of these modes? More importantly, what is the source of energy that drives such a large growth rate for them? We know that the energy cannot derive from inside the disk and so these modes must somehow bring in energy from outside the domain. Extensive investigations of the model indicate that Coriolis and curvature effects are not important to the formation of the wall modes. Consequently, the explanation for their origin and their unphysically high growth rate can be found in a simple Cartesian shear flow model, as described in the next section.

\section{CARTESIAN STUDY OF THE WALL MODES}

In order to gain a better understanding of the mechanism that enhances the growth rate of the wall mode, we have designed the simplest model that exhibits this behavior. We consider an incompressible, ideal, Cartesian shear flow in the presence of a constant applied magnetic field, as shown in Figure 8.

Here the basic state, defined between $z= \pm z_{0}$, is given by

$$
\rho=1, \quad \boldsymbol{U}=U_{0}(z) \boldsymbol{e}_{x}, \quad \boldsymbol{B}=B_{0} \boldsymbol{e}_{y} .
$$

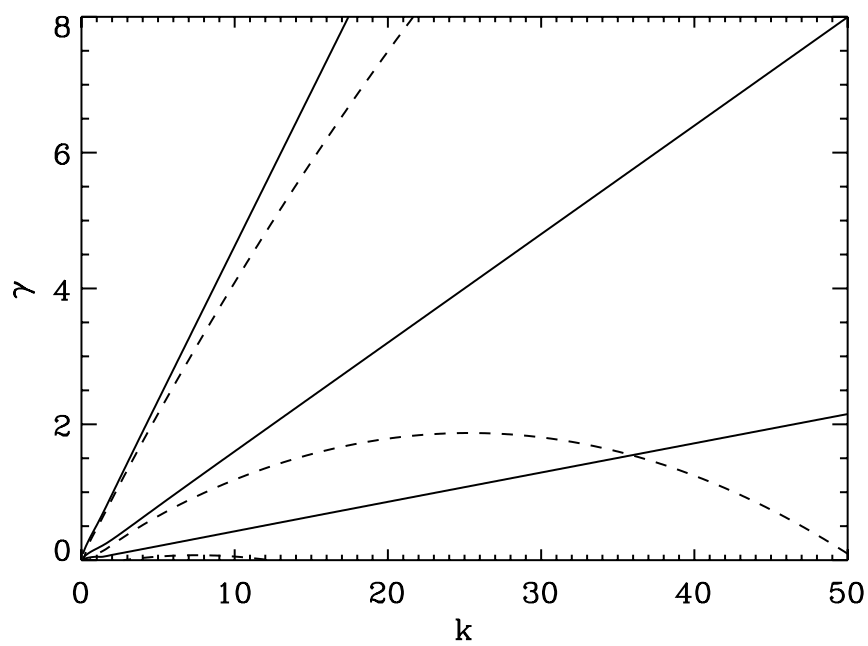

Fig. 7.- Growth rate of the wall mode as a function of $k$ for $m=1$, for $\nu=\eta=0$ (solid lines) and $\nu=\eta=0.001$ (dashed lines), and for $B_{0}=0.025$, 0.1 , and 0.4 , given in increasing slope order. 


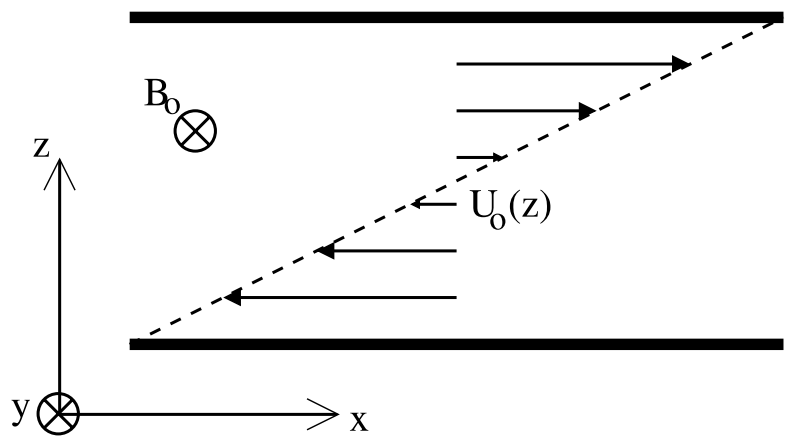

FIG. 8.-Sketch of the configuration of the Cartesian model

We seek perturbations to this basic equilibrium of the form $\boldsymbol{\xi}(\boldsymbol{r}, t)=\boldsymbol{\xi}(z) \exp \left(s t+i k_{x} x+i k_{y} y\right)$, where $\boldsymbol{\xi}(z)$ is the vector of perturbations $\left(u_{x}, u_{y}, u_{z}, b_{x}, b_{y}, b_{z}, \pi\right)$.

Successive substitutions and manipulations of the derived perturbation equations yield the simplified linear system

$$
\begin{aligned}
\chi \mathcal{H} u_{x} & =-U_{0}^{\prime} \mathcal{H} u_{z}-i k_{x} \pi, \\
\chi \mathcal{H} u_{y} & =-i k_{y} \pi, \\
\chi \mathcal{H} u_{z} & =-\pi^{\prime}, \\
0 & =i k_{x} u_{x}+i k_{y} u_{y}+u_{z}^{\prime},
\end{aligned}
$$

where the primes denote differentiation with respect to $z$. Here $\omega_{a}=k_{y} B_{0}$ is the Alfvén frequency and $\chi=s+i k_{x} U_{0}$ is the Doppler-shifted complex growth rate of the mode considered. For simplicity, we have also defined $\mathcal{H}=\left(1+\omega_{a}^{2} / \chi^{2}\right)$; note that the magnetic field appears only in this coefficient.

As yet we have not defined any boundary conditions for this linear system. We shall show once again that the properties of the solutions depend crucially on the choice of boundary conditions. This is the case even in the hydrodynamic limit. If the magnetic field is removed from the basic state, then $\omega_{a}=0$ and $\mathcal{H}=1$, and the system of linear equations given by equation (31) simplifies to

$$
\chi\left[u_{z}^{\prime \prime}-\left(k^{2}+\frac{\chi^{\prime \prime}}{\chi}\right) u_{z}\right]=0,
$$

where $k^{2}=k_{x}^{2}+k_{y}^{2}$.

The importance of the boundary conditions can be seen even for the simplest case of a linear velocity profile. Here $\chi^{\prime \prime}=0$ and the general solution of equation (32) is $u_{z}=c_{-} \exp (-k z)+c_{+} \exp (k z)$. For the usual case of rigid boundary conditions with $u_{z}=0$, clearly no discrete mode can develop (as $c_{ \pm}=0$ trivially) but only a continuum of stable modes, corresponding to $\chi=0$ (see Case 1960). Here we emphasize that discrete modes are smooth, while continuum modes are discontinuous or singular at the point where $\chi=0$. However, if the rigid boundary condition is relaxed in favor of a condition on the velocity tangential to the boundary that allows $u_{z} \neq 0$ there (e.g., $u_{x}=0$ ), then wall modes, which decay exponentially away from the boundaries, become natural solutions of the problem. Furthermore, these modes are marginally stable, i.e., $\gamma=\Re(s)=0$. In this case, because the basic state is symmetric about the midplane $z=0$, the wall modes are also symmetric in $z$.

The inclusion of a magnetic field perpendicular to the shear flow yields the equation

$u_{z}^{\prime \prime}-2 \frac{\omega_{a}^{2}}{\chi^{2}+\omega_{a}^{2}} \frac{\chi^{\prime}}{\chi} u_{z}^{\prime}-\left[k^{2}+\frac{\chi^{\prime \prime}}{\chi}-2 \frac{\omega_{a}^{2}}{\chi^{2}+\omega_{a}^{2}}\left(\frac{\chi^{\prime}}{\chi}\right)^{2}\right] u_{z}=0$

for the velocity $u_{z}$. Figure $9 a$ shows the growth rate $\gamma$ as a function of the applied field strength $B_{0}$ for the case of a linear shear profile with $u_{x}=0$ at the boundaries. The hydrodynamic wall modes, which are marginally stable, remain so for small enough values of $B_{0}$, but as $B_{0}$ is increased, instability to wall mode solutions sets in. These wall modes are stabilized when $B_{0}$ is such that an Alfvén singularity, with $\omega=-k_{x} U_{0} \pm \omega_{a}$, appears somewhere in the domain. The eigenfunctions for the wall modes are shown in Figure $9 b$. As the strength of the magnetic field is increased, a

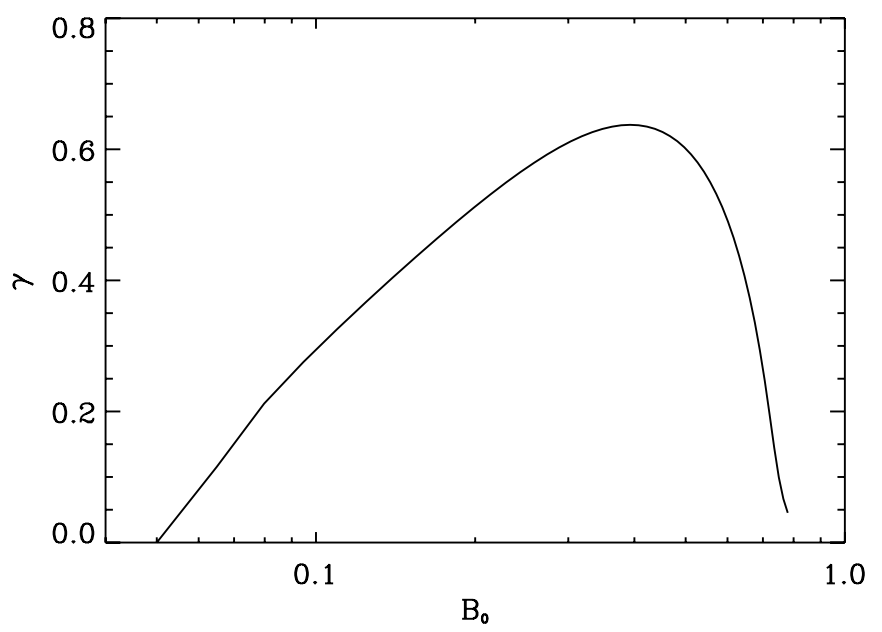

FIG. $9 a$

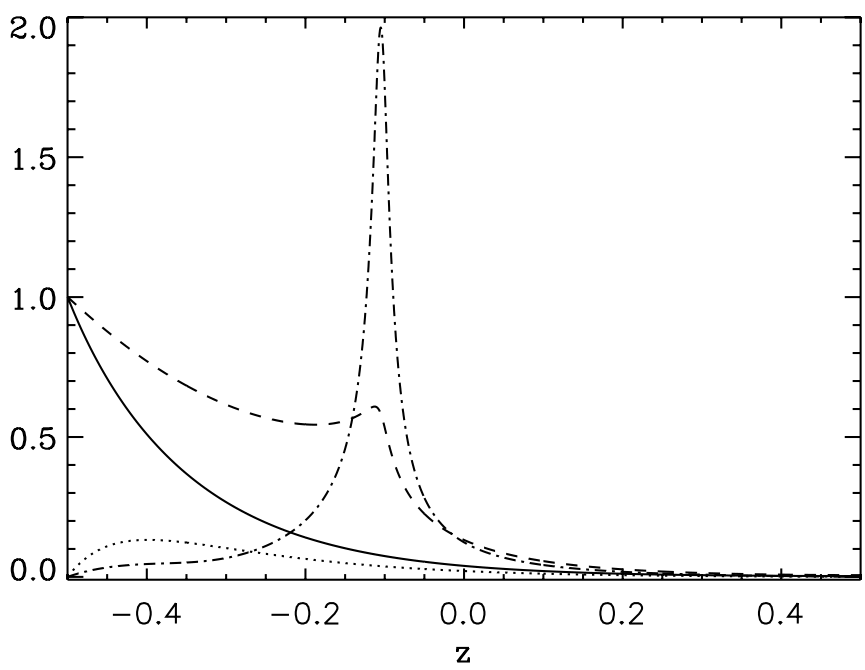

FIG. $9 b$

FIG. 9.- (a) Growth rate of the wall mode as a function of $B_{0}$ for a Cartesian linear shear flow with $k_{x}=k_{y}=4.5$. (b) Eigenfunctions for two values of the magnetic field. The solid and dotted lines show $u_{x}$ and $u_{z}$, respectively, for $B_{0}=0.05$. The dashed and dot-dashed lines represent $u_{x}$ and $u_{z}$, respectively, but for $B_{0}=0.78$, in the vicinity of the Alfvén resonance. 
singularity develops in the eigenfunction at the Alfvén radius, and the mode becomes stabilized. The fact that we do not observe a stabilization with increasing $B_{0}$ of the wall modes in cylindrical geometry leads us to surmise that no such Alfvén singularity occurs in the disk for the parameter values we have studied.

What is the mechanism of the initial destabilization of the wall modes? Clearly the boundary conditions play a crucial role in allowing them to develop. The source of external energy for the instability can be identified using an energy argument, in which the rate of change of total energy is expressed as a sum of surface fluxes involving products of perturbation variables. If $u_{z}=0$, all of these surface terms vanish. However, if $u_{z} \neq 0$, there are many terms that contribute to the flux of energy, any of which could, in principle, enhance the growth of the wall modes. It is not possible (save for having $u_{z}=0$ ) to impose boundary conditions that completely prevent a flux of energy across the boundaries. Thus, to try to reduce the growth rate of the wall modes, we choose boundary conditions that remove some of the surface terms, although there is no guarantee a priori that this will lead to a reduction in the growth rate. To this end we have adopted the boundary condition of setting $\pi=0$ on both boundaries. This is a physically meaningful condition, readily implemented even in the nonlinear regime, and (for the cylindrical model) is consistent with preventing the rundown of the disk.

We have implemented the $\pi=0$ boundary condition for the Cartesian model, with the result that the wall modes are completely stabilized. Clearly this boundary condition has the desired effect of reducing the energy influx to the domain. An analysis of the surface terms also reveals that with $u_{x}=0$ and $U_{0}^{\prime}=0$ on the boundaries, there is no flux of energy into the domain. Numerical investigations confirm that wall modes are indeed stabilized for velocity profiles with vanishing derivative on the boundaries. Further numerical investigations also reveal that the wall modes are stabilized if the applied field $B_{0}$ is zero at the boundaries.

\section{SUPPRESSING THE WALL MODES}

Armed with the insight gained from the Cartesian model of $\S 4$, we are now in a position to address the steps needed to limit the unphysically high growth rate of the wall modes discussed in $\S 3.3$. As one of our primary objectives is to incorporate the accretion process into our model, we must choose boundary conditions that allow $u_{r} \neq 0$ on the boundaries. Given the ar-

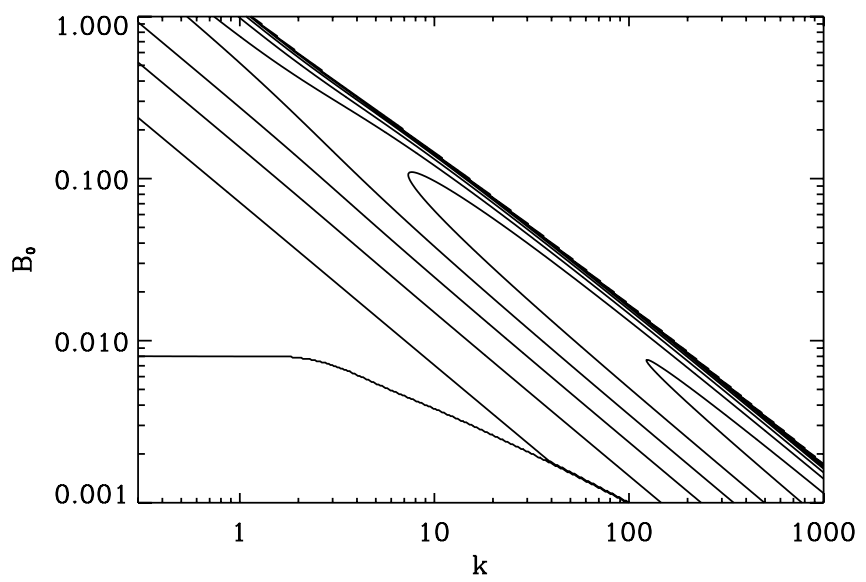

guments of $\S 4$, there remain two options available to us. One approach is to remove the wall modes entirely by modifying the profile of the basic state at the boundaries by setting either the velocity gradient or the magnetic field zero there. Indeed, we have demonstrated that this approach works, but it is not an entirely satisfactory solution to modify the profile of the disk in this way. Furthermore, even if the basic state were so modified, the wall modes might well reappear in the course of the nonlinear evolution. Instead, however, we prefer to restrict the growth rate of any wall modes by modifying the boundary conditions to limit the energy entering the domain from outside. Accordingly, we adopt the boundary condition given by equation (26). With this choice of boundary condition, and in contrast to the Cartesian case, exponentially growing wall modes can still be found attached to either the inner or outer boundaries of the domain. The wall modes that grow along the inner boundary are more unstable, since the shear frequency, which is a measure of the free energy available for the development of local modes, reaches its highest value there.

In contrast to the boundary conditions of $\S 3$, wall modes can now be found even for axisymmetric dissipationless disks (see Fig. 10a). In this case the boundary conditions combined with the equations do not imply that $u_{r}=0$ on the boundary, which would disallow the possibility of wall modes. Moreover, in the nondissipative regime, the wall modes are unstable over a wide range of azimuthal wavenumbers: Figure $10 \mathrm{~b}$ shows the growth rates for a nonaxisymmetric $(m=10)$ wall mode, as a function of $k$ and $B_{0}$. What is encouraging, however, is that the behavior of these wall modes, both axisymmetric and nonaxisymmetric, is entirely consistent with the available free energy of the system. In both cases the maximum growth rate appears to tend to 0.75 as $B_{0} \rightarrow 0$ and $k \rightarrow \infty$ with the product $k B_{0}$ held fixed.

The addition of a small amount of dissipation to the system has the expected effect, as shown in Figure 11. Wall modes are again unstable for axisymmetric and nonaxisymmetric perturbations (although the range of unstable azimuthal wavenumbers is smaller than for the dissipationless case). Dissipation has damped these wall modes and cut off the instability for the highest values of $k$. Again the growth rates of these modes are entirely consistent with the free energy available in the system. Here we do not present the eigenfunctions of the wall modes as their profiles are similar to those shown in Figure 6: it is only the growth rate of the modes that has been substantially reduced.

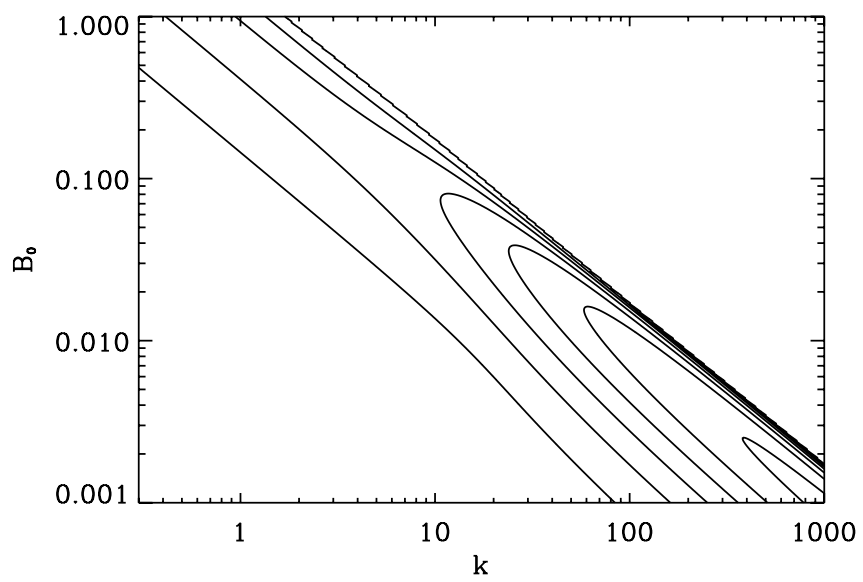

Fig. 10.-Contour plot in $k-B_{0}$ parameter space of the growth rate of the most unstable wall mode in the nondissipative disk. (a) Axisymmetric wall mode; contour values equally spaced between 0.001 and 0.72 . (b) Nonaxisymmetric mode with $m=10$; contour values equally spaced between 0.001 and 0.703 . 


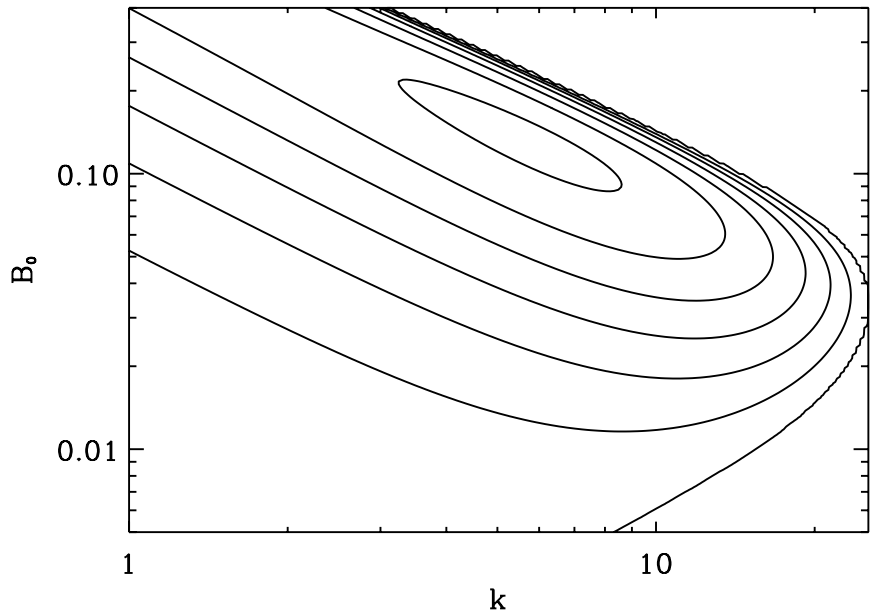

FIG. $11 a$

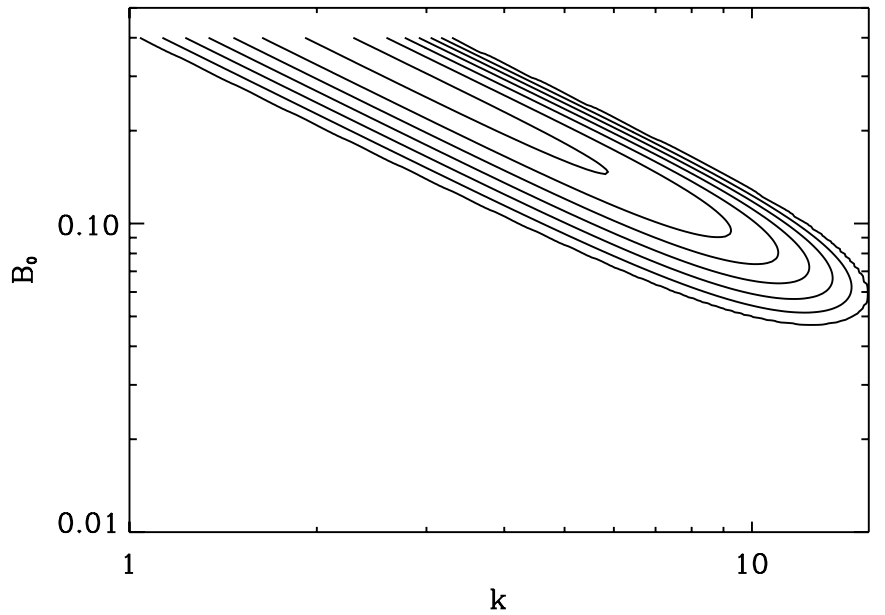

FIG. $11 b$

FIG. 11.-Same as Fig. 10, but for a dissipative disk with $\nu=\eta=0.001$. (a) Axisymmetric wall mode: this mode is nonoscillating with maximal growth rate $\gamma=0.537$; contour levels equally spaced between 0.001 and 0.521 . (b) Nonaxisymmetric $(m=10)$ wall mode with maximal growth rate $\gamma=0.123$ and frequency $\omega=-9.481$; contour levels equally spaced between 0.001 and 0.120 .

The suppression of the growth rate of the wall modes is illustrated clearly in Figure 12, which shows the mode of maximum growth, rate, for both the axisymmetric and nonaxisymmetric wall modes, as a function of the applied field $B_{0}$. The axisymmetric mode has the highest growth rate $\gamma \sim 0.6$ for $B_{0} \sim 0.05$; this remains true for all the values of $B_{0}$ and $k$ considered.

Unsurprisingly, the boundary condition given by equation (26) also allows exponentially growing body modes. The growth rates for the wall modes are now comparable with those for the body modes as shown in Figure 13. For these boundary conditions the fastest growing body mode is axisymmetric with growth rate $\gamma \sim 0.45$.

The new boundary conditions are therefore very successful in limiting the growth rate of the wall modes through prohibiting the influx of energy to the system through the radial surfaces. Although the wall modes are still present for the new set of boundary conditions, they have reasonable growth rates commensurate with the energy available within the differentially rotating disk. It is not unreasonable therefore that these wall modes should exist in conjunction with the unstable body modes in a global model of an accretion disk.

\section{THE ROLE OF AN AZIMUTHAL FIELD}

Our primary aim in this paper has been to investigate the role of boundary conditions on the linear growth of instabilities from a state with a uniform vertical magnetic field, with an eye to formulating appropriate boundary conditions for the nonlinear evolution. In the final evolved state, however, we expect the azimuthal field to dominate, and it is therefore of interest to investigate the significance of wall modes in this case and how they are affected by the choice of boundary conditions.

We have considered a series of equilibria with a uniform vertical field, $B_{z}=B_{0}$, and with the azimuthal field given by equation (15) with $\zeta=0$ and with different values of the constant $B_{1}$. As a check on the new terms introduced by incorporating $B_{\varphi}$, we have recovered the results of Ogilvie \& Pringle (1996) for the case when $B_{0}=0$ and there is no dissipation. Based on the findings of $\S 5$, and for comparison with

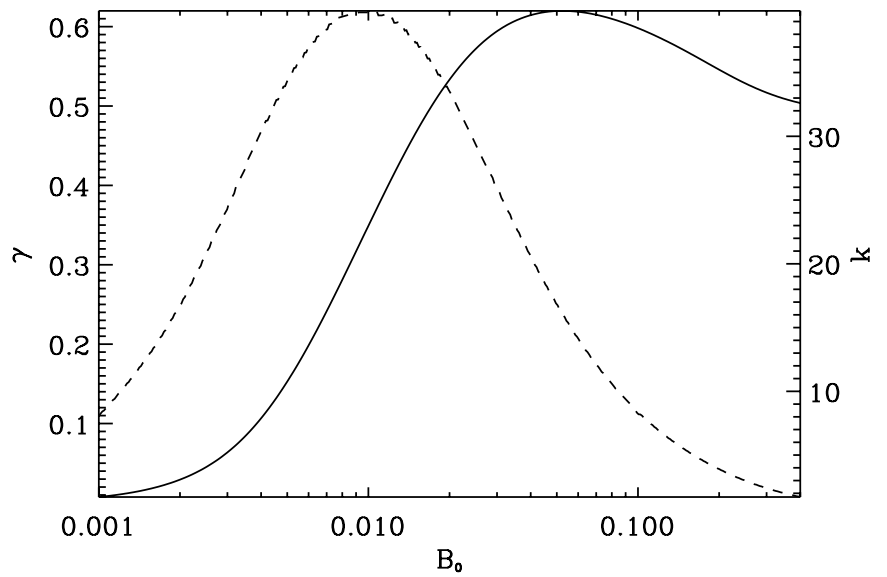

FIG. $12 a$

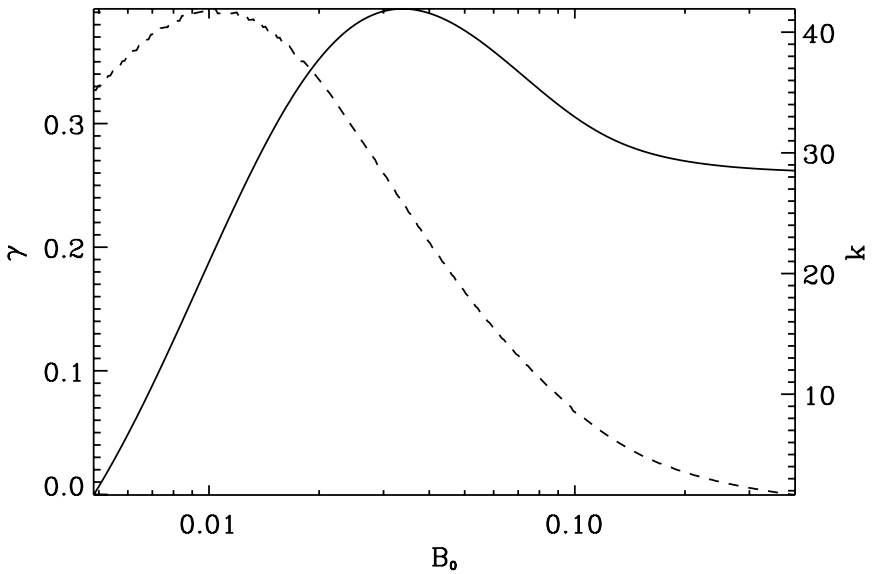

Fig. $12 b$

FIG. 12. - Growth rate (solid line) and corresponding wavenumber (dashed line) of the mode of maximum growth rate as a function of $B_{0}$ for a wall mode with $\nu=\eta=1 \times 10^{-4}$. (a) Axisymmetric wall mode: this is nonoscillating with maximal growth rate $\gamma=0.6199$, obtained for $k=16.0$ and $B_{0}=0.0527$. (b) Nonaxisymmetric $(m=10)$ wall mode with maximal growth rate $\gamma=0.3928$ and frequency $\omega=-9.6839$, obtained for $k=25.7$ and $B_{0}=0.0341$. 


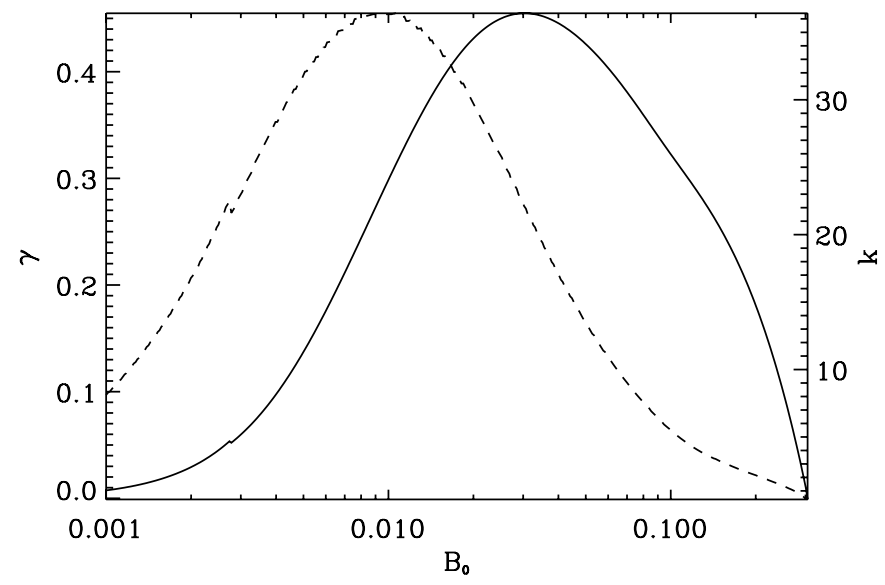

FIG. 13.- Same as Fig. 12, but for an axisymmetric body mode, with maximal growth rate $\gamma=0.4548$, obtained for $k=22.1$ and $B_{0}=0.0302$.

the results obtained for a purely vertical field, we have employed the linearized boundary conditions given by equations (24) and (26) (although it should be noted that the equilibrium azimuthal field does not satisfy eq. [21]). In keeping with the results for a purely vertical field, we find that with an additional azimuthal component this choice of boundary conditions yields growth rates of the wall modes consistent with the local properties of the disk. On fixing $B_{0}$ and increasing $B_{1}$, we find little change in the eigenvalues and eigenfunctions of the unstable modes until $B_{1}$ exceeds $B_{0}$ (see Fig. 14), with the wall modes having the larger growth rate. As the influence of the azimuthal field becomes significant, the growth of both types of mode is reduced. Interestingly, the wall modes are suppressed more strongly, leading, for a small range of $B_{1}$, to the body modes becoming dominant. For sufficiently high $B_{1}$ all instabilities are suppressed. It can be seen from Figure 14, which shows the growth rates of axisymmetric modes, that as the influence of the azimuthal field increases, the preferred vertical wavenumber $k$ decreases. In a similar manner, increasing the azimuthal field leads to a reduction in the wide range of unstable $m$ wavenumbers for nonaxisymmetric wall modes.

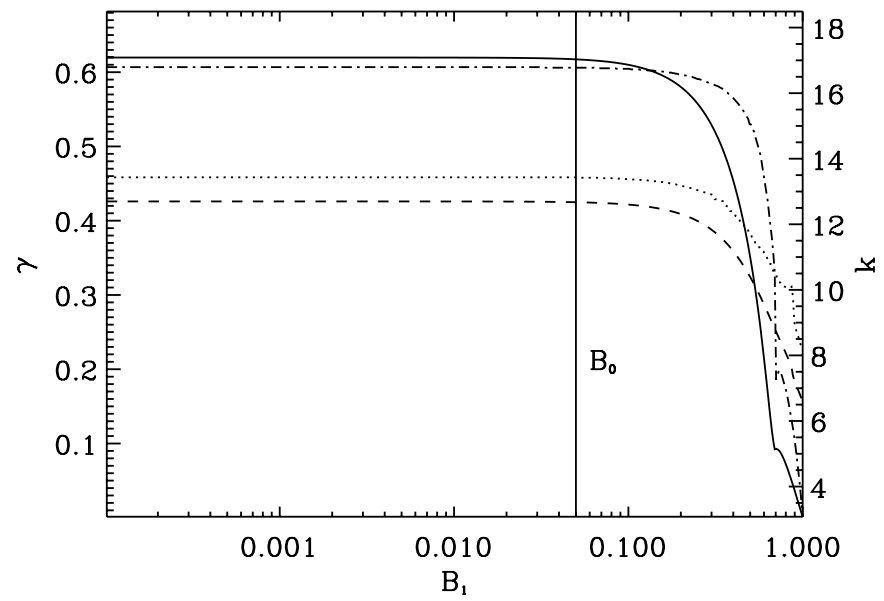

FIG. 14.-Growth rates and vertical wavenumbers of the most unstable (axisymmetric) wall mode and body mode as a function of $B_{1}$ with $B_{0}=0.05$. The solid and dashed lines denote the growth rates of the wall mode and body mode, respectively; the dot-dashed and dotted lines refer to the corresponding wavenumbers.

\section{CONCLUSIONS}

In this paper we have examined linear MRIs in a global model of a hydromagnetic incompressible accretion disk designed to allow accretion in both the basic and fully nonlinear states. We have demonstrated that for a self-consistent global model with accretion, the radial boundary conditions must be selected with extreme care as they have a significant bearing on the form of the solutions even in the linear regime. For all boundary conditions that allow accretion $\left(u_{r} \neq 0\right.$ at the radial boundaries), wall modes exist in conjunction with the usual body modes. Indeed, if the boundary conditions are not chosen carefully, then these wall modes have growth rates that are too large for the free energy available from the shear flow. The energy source for these wall modes was identified in $\S 4$ by considering a simple Cartesian shear flow analog and identifying the terms that contribute to the large growth rate of the mode. This simplified model suggested an improved set of boundary conditions (with constant total pressure at the radial boundaries) that was found to reduce the growth rates of both axisymmetric and nonaxisymmetric wall modes to levels similar to those found for global body modes in the disk. It is worth noting that experimental investigations of the MRI must, of necessity, employ a no-slip condition on the radial boundaries (i.e., eq. [17] together with $u_{r}=0, u_{z}=0$ ); as such, and in keeping with the stability analyses of Goodman \& Ji (2002) and Noguchi et al. (2002), we do not anticipate the appearance of wall modes in these systems.

These results are important for global models of disks. At present, many global disk models prescribe a finite initial amount of matter in the disk (e.g., Hawley 2000 and subsequent works), and the nonlinear evolution of the disk constitutes a run-down experiment as the matter is accreted at the inner boundary or lost through the other boundaries. For these models it is not possible to reach a statistically steady hydromagnetic state as the disk is isolated at the exterior boundary from its surroundings. Other global models (e.g., Willis \& Barenghi 2002; Drecker, Rüdiger, \& Hollerbach 2000) consider boundary conditions that do not allow accretion either into or out of the computational domain. For these models a statistically steady state is possible, but no net accretion is allowed. The model we consider here allows both accretion and relaxation to a statistically steady state. However, as we have demonstrated, in such a model, it is important to apply appropriate boundary conditions at the radial boundaries.

Work is in progress on nonlinear extensions for this global model. The saturation mechanism for these instabilities is of obvious interest in both two and three dimensions. In three dimensions there is also the possibility of obtaining a selfsustaining, bootstrapping process for which the field is responsible for the generation of the turbulence that feeds the dynamo. The nature of saturation mechanisms for dynamos is a topic of considerable astrophysical interest, and further global modeling will aid our understanding of the complicated nonlinear interactions at work in the equilibration of the magnetic field.

The authors acknowledge financial support from PPARC under grant PPA/G/S/2000/00147. We are also grateful to the anonymous referee for comments that have improved the presentation of the manuscript. 


\section{APPENDIX}

\section{THE GOVERNING LINEAR EQUATIONS}

The basic equilibrium state that we have considered has nonzero radially dependent values of the radial and azimuthal velocities, $U_{r} \sim r^{-1}$ and $U_{\varphi}=r \Omega \sim r^{-1 / 2}$, and, most generally, a magnetic field of the form $\boldsymbol{B}=\left[0, B_{\varphi}(r), B_{z}(r)\right]$, where $B_{\varphi}$ and $B_{z}$ satisfy the equilibrium conditions given by equations (14) and (15). On assuming a normal mode solution, in which all variables take the form $\xi(r) \exp (s t+i m \varphi+i k z)$, and denoting the Eulerian perturbations to the velocity and magnetic field as $\boldsymbol{u}=\left(u_{r}, u_{\varphi}, u_{z}\right)$ and $\boldsymbol{b}=\left(b_{r}, b_{\varphi}, b_{z}\right)$, respectively, and the perturbation to the total pressure as $\pi$, the equations governing the linear perturbations may be expressed as

$$
\begin{aligned}
& s u_{r}=-\left\{-\frac{U_{r}}{r}+\frac{\nu}{r^{2}}\left[m^{2}+(k r)^{2}+1\right]+i m \Omega\right\} u_{r}-\left(U_{r}-\frac{\nu}{r}\right) u_{r}^{\prime}+\nu u_{r}^{\prime \prime}+\left(2 \Omega-2 i m \frac{\nu}{r^{2}}\right) u_{\varphi}-\pi^{\prime}+i\left(m \frac{B_{\varphi}}{r}+k B_{z}\right) b_{r}-2 \frac{B_{\varphi}}{r} b_{\varphi}, \\
& s u_{\varphi}=\left(-\frac{\Omega}{2}+2 i m \frac{\nu}{r^{2}}\right) u_{r}-\left\{\frac{U_{r}}{r}+\frac{\nu}{r^{2}}\left[m^{2}+(k r)^{2}+1\right]+i m \Omega\right\} u_{\varphi}-\left(U_{r}-\frac{\nu}{r}\right) u_{\varphi}^{\prime}+\nu u_{\varphi}^{\prime \prime}-\frac{i m}{r} \pi+\left(\frac{B_{\varphi}}{r}+B_{\varphi}^{\prime}\right) b_{r}+i\left(m \frac{B_{\varphi}}{r}+k B_{z}\right) b_{\varphi} \\
& s u_{z}=-\left\{\frac{\nu}{r^{2}}\left[m^{2}+(k r)^{2}\right]+i m \Omega\right\} u_{z}-\left(U_{r}-\frac{\nu}{r}\right) u_{z}^{\prime}+\nu u_{z}^{\prime \prime}-i k \pi+B_{z}^{\prime} b_{r}+i\left(m \frac{B_{\varphi}}{r}+k B_{z}\right) b_{z}, \\
& 0=\frac{1}{r} u_{r}+u_{r}^{\prime}+\frac{i m}{r} u_{\varphi}+i k u_{z} \\
& s b_{r}=i\left(\frac{m}{r} B_{\varphi}+k B_{z}\right) u_{r}-\left\{\frac{U_{r}}{r}+\frac{\eta}{r^{2}}\left[m^{2}+(k r)^{2}+1\right]+i m \Omega\right\} b_{r}-\left(U_{r}-\frac{\eta}{r}\right) b_{r}^{\prime}+\eta b_{r}^{\prime \prime}-2 i m \frac{\eta}{r^{2}} b_{\varphi}, \\
& s b_{\varphi}=\left(\frac{B_{\varphi}}{r}-B_{\varphi}^{\prime}\right) u_{r}+i\left(\frac{m}{r} B_{\varphi}+k B_{z}\right) u_{\varphi}+\left(-\frac{3}{2} \Omega+2 i m \frac{\eta}{r^{2}}\right) b_{r}-\left\{-\frac{U_{r}}{r}+\frac{\eta}{r^{2}}\left[m^{2}+(k r)^{2}+1\right]+i m \Omega\right\} b_{\varphi}-\left(U_{r}-\frac{\eta}{r}\right) b_{\varphi}^{\prime}+\eta b_{\varphi}^{\prime \prime} \\
& s b_{z}=-B_{z}^{\prime} u_{r}+i\left(\frac{m}{r} B_{\varphi}+k B_{z}\right) u_{z}-\left\{\frac{\eta}{r^{2}}\left[m^{2}+(k r)^{2}\right]+i m \Omega\right\} b_{z}-\left(U_{r}-\frac{\eta}{r}\right) b_{z}^{\prime}+\eta b_{z}^{\prime \prime} .
\end{aligned}
$$

\section{REFERENCES}

Armitage, P. J. 1998, ApJ, 501, L189

Balbus, S. A., \& Hawley, J. F. 1998, Rev. Mod. Phys., 70, 1

Blandford, R. D., \& Payne, D. G. 1982, MNRAS, 199, 883

Brandenburg, A., Nordlund, A., Stein, R. F., \& Torkelsson, U. 1995, ApJ, 446, 741

Case, K. M. 1960, Phys. Fluids, 3, 143

Curry, C., \& Pudritz, R. E. 1996, MNRAS, 281, 119

Drecker, A., Rüdiger, G., \& Hollerbach, R. 2000, MNRAS, 317, 45

Dubrulle, B., \& Knobloch, E. 1993, A\&A, 274, 667

Fleming, T., \& Stone, J. M. 2003, ApJ, 585, 908

Gammie, C. F., \& Balbus, S. A. 1994, MNRAS, 270, 138

Goldreich, P., \& Lynden-Bell, D. 1965, MNRAS, 130, 97

Goodman, J., \& Ji, H. 2002, J. Fluid Mech., 462, 365

Hawley, J. F. 2000, ApJ, 528, 462

Hawley, J. F., Gammie, C. F., \& Balbus, S. A. 1995, ApJ, 440, 742 1996, ApJ, 464, 690

Knobloch, E. 1992, MNRAS, 255, 25P

Lynden-Bell, D., \& Pringle, J. E. 1974, MNRAS, 168, 603

Machida, M., Hayashi, M. R., \& Matsumoto, R. 2000, ApJ, 532, L67

Matsumoto, R., \& Tajima, T. 1995, ApJ, 445, 767
Miller, K. A., \& Stone, J. M. 2000, ApJ, 534, 398

Moffatt, H. K. 1978, Magnetic Field Generation in Electrically Conducting Fluids (Cambridge: Cambridge Univ. Press)

Narayan, R., Quataert, E., Igumenshchev, I. V., \& Abramowicz, M. A. 2002, ApJ, 577, 295

Noguchi, K., Pariev, V. I., Colgate, S. A., Beckley, H. F., \& Nordhaus, J. 2002, ApJ, 575, 1151

Ogilvie, G. I., \& Pringle, J. E. 1996, MNRAS, 279, 152

Papaloizou, J. C. B., \& Nelson, R. P. 2003, MNRAS, 339, 983

Sano, T., \& Stone, J. M. 2002, ApJ, 577, 534

Shibata, K., \& Uchida, Y. 1985, PASJ, 37, 31 . 1986, PASJ, 38, 631

Steinacker, A., \& Papaloizou, J. C. B. 2002, ApJ, 571, 413

Stone, J. M., Hawley, J. F., Gammie, C. F., \& Balbus, S. A. 1996, ApJ, 463, 656

Tagger, M., \& Pellat, R. 1999, A\&A, 349, 1003

Terquem, C., \& Papaloizou, J. C. B. 1996, MNRAS, 279, 767

Turner, N. J., Stone, J. M., \& Sano, T. 2002, ApJ, 566, 148

Willis, A. P., \& Barenghi, C. F. 2002, A\&A, 388, 688 